\title{
Bubble Pump Theoretical Model with the Assumption of Laminar Flow Regime
}

\author{
Bella Gurevich \\ Shamoon College of Engineering, Mechanical Engineering Department \\ Jabotinsky 24, Ashdod, Israel \\ bellagu@sce.ac.il
}

\begin{abstract}
The bubble pump is the main part in diffusion absorption cooling systems where heating, pumping of the binary solution and the separation occurs. Up to date the existing theoretical models of the bubble pump where initially developed for air lift pumps where neither heating nor separation occurs. Thus, the experimental results that were conducted for a bubble pump did not correlate well with the theoretical models. Empirical values were suggested in some of the models, however, their values varied from one system to another and could not be predicted analytically. In this work a modified model based on mass, energy, momentum and heat balances is presented. with the utilization of the drift flux model with laminar flow assumption is presented. In addition, for the first time the applied heat is expressed in the model. The suggested model fits better with the experimental results than the previous models.
\end{abstract}

Keywords: Diffusion Absorption Cooling Systems, Bubble Pump, Drift Flux Model.

\section{Nomenclature}

A

$C_{o}$

$c_{p}$

$$
\left[\frac{k g}{k g \cdot{ }^{o} \mathrm{C}}\right]
$$

$\mathrm{D}$

$\mathrm{f}$

$\mathrm{g}$

$\mathrm{H}$

$\mathrm{L}$

$\dot{m}$

$\mathrm{P}$

$\operatorname{Re}$

$\mathrm{T}$

$\mathrm{U}$

W

Z

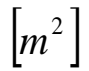

$[\mathrm{m}]$

$\left[\frac{m}{\sec ^{2}}\right]$

$[m]$

$[m]$

$\left[\frac{k g}{\mathrm{sec}}\right]$

$$
[\mathrm{Pa}]
$$

$$
\left[{ }^{\circ} \mathrm{C}\right],[\mathrm{K}]
$$

$$
\left[\frac{m}{\mathrm{sec}}\right]
$$

$[W]$

$[m]$
Pipe cross section

Distribution parameter

Heat capacity

Pipe diameter

Friction coefficient

Gravity

Motive head

Pipe length

Mass flow rate

Pressure

Reynolds number

Temperature

velocity

Heat

Generator height 


\section{Greek symbols}

$$
\alpha
$$

$\mu$

$$
\left[\frac{k g}{m \cdot \sec }\right]
$$

$$
\xi
$$

$\rho$

\section{Subscripts}

$0,1,2,3$
$\mathrm{DC}$
$\mathrm{f}$
$\mathrm{G}$
gen
$\mathrm{L}$
$\mathrm{LT}$
$\mathrm{m}$
poor
ref
res
rich
sys
$\mathrm{TP}$

\author{
Void fraction \\ Viscosity \\ Mass concentration \\ Density
}

\author{
Points $0,1,2,3$ \\ Down comer \\ Friction \\ Gas \\ Generator \\ Liquid \\ Lift tube \\ mixture \\ Poor solution \\ Refrigerant \\ reservoir \\ Rich solution \\ System \\ Two phase
}

\section{Introduction}

Diffusion absorption cooling systems are heat driven and contain no moving parts such as a compressor or a pump. The working fluid is a mixture of a coolant and a solvent together with an inert gas. The main part of such systems is the bubble pump where heating, pumping of the solution and the separation occurs. The bubble pump is a heated tube (length $\mathrm{L}$ and diameter D) connecting two reservoirs; one situated higher than the other (Fig. 1). Liquid in the lower reservoir initially fills the tube to the same level as in the lower reservoir $(\mathrm{H})$. Heat is applied at the bottom of the bubble pump and causes the vapor bubbles to rise in the lifting tube. The bulk density of the fluid in the tube is reduced relatively to that in the lower reservoir, thereby creating an overall buoyancy lift. At the upper reservoir, the gas is separated from the liquid solution. In diffusion absorption refrigeration (DAR) systems the bubble pump is the responsible for the circulation of the binary solution. The solution contains the refrigerant, which acts as a coolant and an organic compound, which acts as the absorbent. DAR systems have COP values that vary in range of $0.1-0.15$.

Throughout the years since its first presentation by Platen and Munters in 1921 and later on by Einstein and Szilard in 1930, theoretical models were developed to predict the performance of DAR systems focusing on vapor lift pumps. There are not many works in the literature that cover the bubble pump. More covered topic in the literature is the Air-Lift pump that operates with the same principals as vapor-lift pumps with the exception of the air being injected to increase the buoyancy of the fluid instead of bubbles forming from liquid vaporization.

Analytical treatment of air-lift pumps based on two-phase slug flow theory was developed later by [1]. In his study, [1] defined the efficiency of the pump as the work done in lifting the liquid divided by the work done by the air as it expands isothermally.

First were [2] who developed an analytical model using one dimensional mass and momentum equations together with the basic equations of two-phase flow taking in consideration the effects of friction and slip between the gas and liquid phases (drift flux model). Liquid volume flow rates were plotted versus air volume flow rates for various submergence ratios $(\mathrm{H} / \mathrm{L})$. 
A comparison with experimental work was carried out, and the authors predicted that the theory of one dimensional flow is sufficient for the analysis of airlift pump performance.

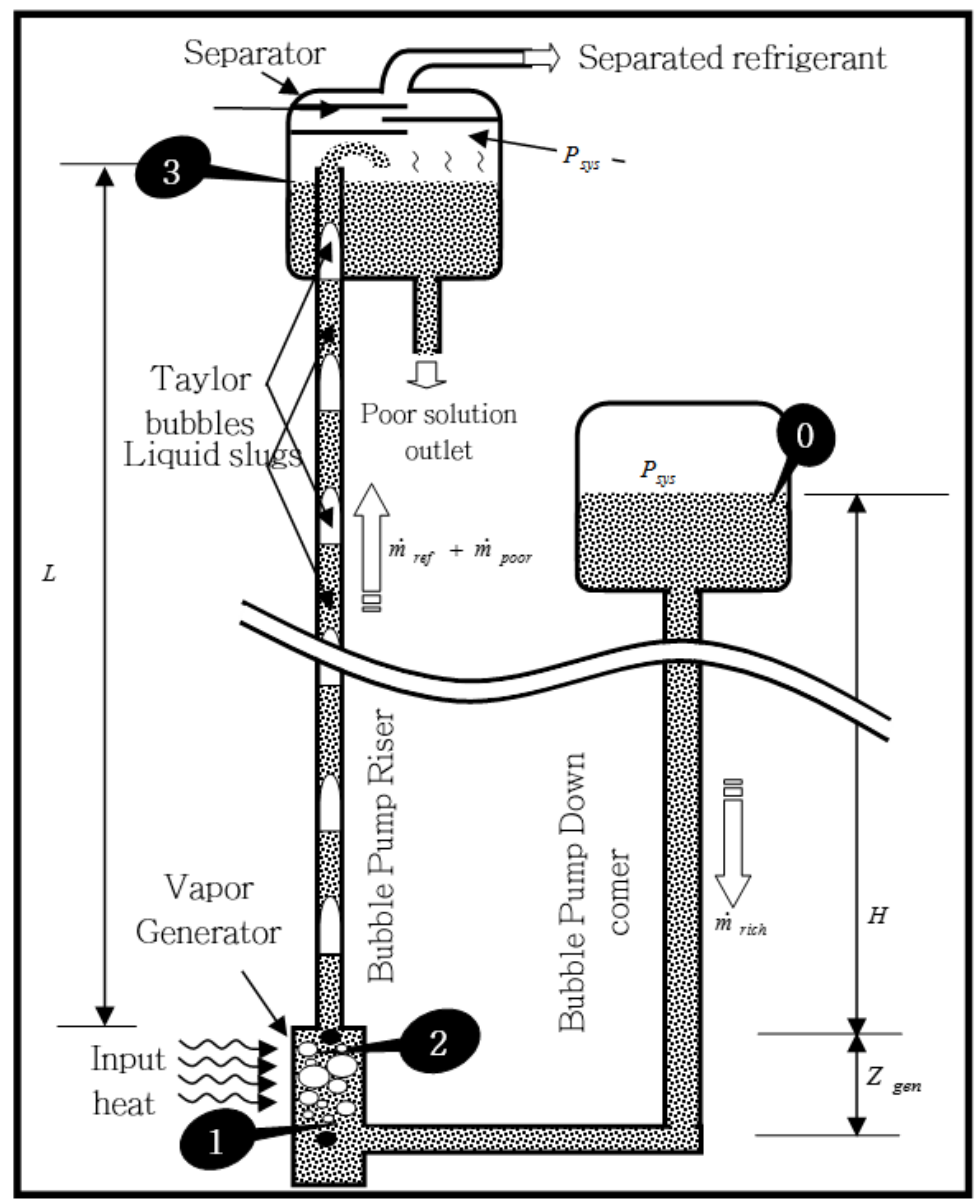

Fig. 1: Schematic drawing of the bubble pump.

[3] studied theoretically and experimentally the vapor bubble pump used in the Einstein diffusion Vapor Absorption Refrigeration System. The [2] model was firstly modified to analyse the performance of the bubble pump. Instead of using the drift flux model to find the void fraction, Delano assigned a value to the slip, S.

[4] studied theoretically and experimentally the bubble pump performance assuming that the flow in the vertical lift tube was laminar and using $\mathrm{K}$ adjustable parameter to account for losses other than friction in the tube. The mass flow rate of vapor increased linearly with the heat input whereas the mass flow rate of the pumped liquid first increased, reached a maximum value and then decreased with the increase in the heat input. The system was best operated at the maximum liquid mass flow rate when the efficiency was the highest. Larger diameter pump tube would always be advantageous. However, increasing the diameter with a fixed liquid flow eventually caused transition from the assumed slug flow([5]) to bubbly flow.

[6] carried out an experimental investigation on a closed continuous bubble pump experimental system. The working fluids were organic solvent as absorbent and hydro-chlorofluorocarbon (R22) as a refrigerant. The results showed that the bubble pump operated at the slug flow regime with a churn flow regime at the entrance to the bubble pump tube.

[7] performed a theoretical and experimental study on the vapor bubble pump. The Delano's model (1998) was modified and the method that was recommended by [2] was developed to evaluate the frictional pressure drop. Theoretically, it is found that the length of the riser beyond $1.3 \mathrm{~m}$ had insignificant effect on its performance. The theoretical prediction of the pumping capacities was lower than the experimental results for all values of tube diameter and submergence ratios. A new K-factor equation was introduced to correlate the theoretical result with the experimental data. 
The work of [8] constructed an experimental continuous system that aimed to investigate the performance of three parallel bubble pumps with a solution of R134a-DMAC. The dependence of the number of bubble pump lift tubes and various operating conditions on the amount of the desorbed refrigerant was examined. The results showed that in comparison to a single lift tube, the use of two or three parallel lift tubes at optimal heat input and mass concentration could double or triple, correspondingly, the amount of desorbed refrigerant. There was not a good correlation with the theoretical model of [3]

The current models are not accurate enough to predict the experimental results. Empirical values are used, however, these values vary from one system to another.

\section{Theoretical Model}

The present model is aimed to predict the amounts of separated refrigerant for a given submergence ratio and rich solution. $m_{\text {ref }}, m_{\text {rich }}, m_{\text {poor }}$ are the mass flow rates of the refrigerant, the rich solution and the poor solution respectively. Referring to the fig. 1, applying the mass balance equation from point 0 to point 1 (Fig. 1):

$$
\dot{m}_{o}=\dot{m}_{1}=\dot{m}_{\text {rich }}
$$

$\dot{m}_{r i c h}$ is the mass flow rate of the rich solution containing the solvent and the refrigerant. Assuming that its value doesn't change along the section $0-1$.

Appling Bernoulli equation from the liquid surface (o) at the down comer to point 1 yields:

$$
\frac{P_{0}}{\rho_{0} g}+\frac{U_{0}^{2}}{2 g}+\left(H+Z_{g e n}\right)=\frac{P_{1}}{\rho_{1} g}+\frac{U_{1}^{2}}{2 g}+f \frac{\left(H+Z_{g e n}\right)}{D_{D C}} \frac{U_{1}^{2}}{2 g}
$$

When $P_{0}$ is the system pressure, the sub index DC refers to the down comer. Assuming that the level of the solution in the reservoir is maintained constant:

$$
U_{0}=0
$$

The velocity of the fluid in section (1) is:

$$
U_{1}=\frac{\dot{m}_{r i c h}}{\rho_{r i c h} \cdot A}
$$

Thus the pressure difference between sections (1) and (0) is in the form of:

$$
\left.P_{\text {sys }}-P_{1}=\frac{\dot{m}_{\text {rich }}^{2}}{2 \rho_{\text {rich }} \cdot A_{D C}{ }^{2}} \mid 1+f\left(\frac{H+Z_{\text {gen }}}{D_{D C}}\right)\right] \mid-\rho_{\text {rich }} g\left(H+Z_{\text {gen }}\right)
$$

Mass conservation and momentum equation are applied for the generator, point 1 for the entrance to the generator and point 2 for the outlet of the generator. Let us assume that at the outlet of the generator there is a two phase flow consisting of the evaporated refrigerant as the gas phase and the poor solution containing the absorbent with residues of refrigerant as the liquid phase. The sub index gen refers to the generator. 


$$
\begin{gathered}
\dot{m}_{1}=\dot{m}_{\text {rich }} \\
\dot{m}_{2}=\dot{m}_{\text {poor }}+\dot{m}_{\text {ref }} \\
\dot{m}_{\text {rich }}=\dot{m}_{\text {poor }}+\dot{m}_{\text {ref }} \\
\dot{m} \cdot\left(U_{2}-U_{1}\right)=\left(P_{1}-P_{2}\right) \cdot A_{g e n}-\rho_{T P} \cdot g \cdot Z_{g e n} \cdot A_{g e n}-\tau_{w} \cdot \pi D Z_{g e n}
\end{gathered}
$$

Thus the pressure drop along the generator:

$$
P_{1}-P_{2}=\frac{\dot{m}_{r i c h}}{A_{g e n}} \cdot\left(U_{2}-U_{1}\right)+\rho_{T P} \cdot g \cdot Z_{g e n}+f_{T P} \rho_{I P} \frac{1}{2}\left(\frac{\dot{m}_{r i c h}}{A_{g e n}}\right)^{2} \frac{Z_{g e n}}{D_{g e n}}
$$

Since the generator is relatively a short tube, gravitational and frictional pressure drops can be neglected:

$$
P_{1}-P_{2}=\frac{\dot{m}_{r i c h}}{A_{g e n}} \cdot\left(U_{2}-U_{1}\right)
$$

The area of interest is the lifting tube. Therefore, mass and momentum balances are applied between points 2 and 3:

Let us assume that the mass flows rates of the refrigerant and the poor solution are constant along the lift tube. The sub index LT refers to the lift tube.

$$
\begin{gathered}
\dot{m}_{2}=\dot{m}_{3} \\
\dot{m}_{\text {poor }, 2}+\dot{m}_{r e f, 2}=\dot{m}_{\text {poor }, 3}+\dot{m}_{r e f, 3}=\dot{m}_{\text {poor }}+\dot{m}_{r e f} \\
\dot{m} \cdot\left(U_{3}-U_{2}\right)=\left(P_{3}-P_{2}\right) \cdot A_{L T}-\rho_{T P} \cdot g \cdot L \cdot A_{L T}-\tau_{w} \cdot A_{L T}
\end{gathered}
$$

At point 3 the pressure is assumed to be system pressure. The overall pressure drop along the lift tube can be expressed as a function of acceleration pressure drop, gravitational pressure drop and frictional pressure drop.

$$
P_{2}-P_{s y s}=\frac{\dot{m}}{A_{L T}} \cdot\left(U_{3}-U_{2}\right)+\rho_{T P} \cdot g \cdot L+f_{T P} \rho_{T P} \frac{1}{2}\left(\frac{\dot{m}_{r i c h}}{A_{L T}}\right)^{2} \frac{L}{D_{L T}}=\Delta P_{a c c}+\Delta P_{g}+\Delta P_{f}
$$

Assuming that the velocities of the two phases $U_{2}$ and $U_{3}$ along the lift tubes do no vary, thus the accelerational pressure drop can be neglected. the expression for the pressure drop can be written in the following manner:

$$
P_{2}-P_{s y s}=\Delta P_{g}+\Delta P_{f}
$$

When the two phases are considered to have different velocities (e.g., liquid and gas), the relation between void fraction and quality is not analytically calculable, but requires some empirical data which links void and quality. A large number of empirical and semi-empirical methods have been suggested over the last fifty years. The semi-empirical model which seems to have the most physical basis is the drift flux model. It relates the gas-liquid velocity difference to the drift flux (or 'drift 
velocity') of the vapor relative to the liquid; e.g., due to buoyancy effects. This model has been principally developed by [9], [10 and [11].

The gravitational term $\left(\Delta P_{g r a}\right)$ represents the weight of the mixture column in the lifting tube.

$$
\Delta P_{g}=\rho_{T P} \cdot g \cdot L
$$

The density of the solution is calculated by using drift flux model. The drift-flux approach is based on the consideration of two fluids as a mixture in which properties are represented as an average of the properties of the two fluids. The slug mixture density, $\rho_{T P}$, is defined as the average of the liquid and gas phases densities weighted by the void fraction $\alpha$ :

$$
\rho_{T P}=(1-\alpha) \rho_{L}+\alpha \rho_{G}
$$

The one dimensional drift flux model assumes that the two phase flow properties do not change across the pipe cross section, and hence yield the values that are averaged across the pipe cross section for any given axial pipe location. The void fraction in this model is defined as a ratio between the gas phase superficial velocity $U_{G S}$ and the gas phase velocity which can be estimated by the drift kinematic law proposed [9]. $C_{0}$ is the distribution parameter, $U_{m}$ is the average mixture velocity and $U_{G M}$ is the local drift velocity:

$$
\begin{gathered}
\alpha=\frac{U_{G S}}{C_{0} U_{m}+U_{G M}} \\
U_{G S}=\frac{Q_{G}}{A} \\
U_{m}=\frac{Q_{L}+Q_{G}}{A}
\end{gathered}
$$

In the open literature, there are several correlations to calculate the distribution parameter and the local drift velocity. For laminar flows $C_{0} \approx 2$ ([1], [12], [13],[14])

According to [15] the local drift velocity is:

$$
U_{G M}=0.35 \sqrt{\frac{g D\left(\rho_{L}-\rho_{G}\right)}{\rho_{L}}}
$$

The friction pressure drop term takes into account the viscous forces and forces between the wall and fluids and is given by:

$$
\Delta P_{f}=f_{T P} \frac{U_{m}^{2} \rho_{T P}}{2} \frac{L}{D}
$$

Assuming laminar flow:

$$
f_{T P}=\frac{64}{\operatorname{Re}_{m}}
$$


When Reynolds number is defined as:

$$
\operatorname{Re}_{m}=\frac{U_{m} \rho_{T P} D}{\mu_{T P}}
$$

$\mathrm{D}$ is the inner diameter, $U_{m}$ is the total average superficial velocity, $\rho_{T P}$ and $\mu_{T P}$ are the density and the dynamic viscosity of the two phase solution.

By summing equations (5), (9) and (13) and:

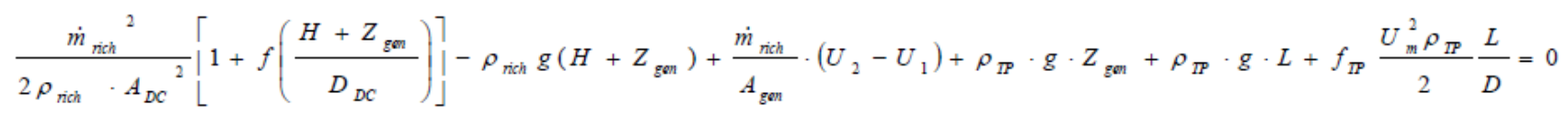

Assuming that:

$$
\begin{gathered}
U_{1}=\frac{\dot{m}_{r i c h}}{A \cdot \rho_{\text {rich }}} \\
U_{2}=U_{m}=\frac{\dot{m}_{\text {poor }}}{A \cdot \rho_{\text {poor }}}+\frac{\dot{m}_{r e f}}{A \cdot \rho_{r e f}}
\end{gathered}
$$

The density and dynamic viscosity of a binary solution at equilibrium can be calculated based on ([16] ) correlation:

$$
\begin{gathered}
\rho=\sum_{j=0}^{3} \sum_{i=0}^{3} \rho_{i, j} \cdot \xi^{i} \cdot T^{j} \\
\ln (\mu)=\sum_{j=0}^{3} \sum_{i=0}^{3} \mu_{i, j} \cdot \xi^{i} \cdot T^{j}
\end{gathered}
$$

Substituting the expressions in equations (24) and (25) into equation (23):

$$
\begin{aligned}
& \frac{\dot{m}_{\text {rich }}^{2}\lceil}{2 \rho_{\text {rich }} \cdot A^{2}}\left[1+f\left(\frac{H+Z_{g e n}}{D}\right)\right]-\rho_{\text {rich }} g\left(H+Z_{g e n}\right)+\frac{\dot{m}_{r i c h}^{2}}{A^{2}} \cdot\left(\frac{1}{\rho_{I P}}-\frac{1}{\rho_{\text {rich }}}\right)+\ldots . . \\
& \ldots+\rho_{T P} \cdot g \cdot\left(Z_{g e n}+L\right)+f_{I P}\left(\frac{\dot{m}_{\text {poor }}}{A \cdot \rho_{\text {poor }}}+\frac{\dot{m}_{\text {ref }}}{A \cdot \rho_{\text {ref }}}\right)^{2} \frac{\rho_{I P}}{2} \frac{L}{D}=0
\end{aligned}
$$

The frictional pressure drop in the down comer and in the lift tube can be neglected:

$$
\frac{\dot{m}_{\text {rich }}{ }^{2}}{2 \rho_{\text {rich }} \cdot A_{D C}{ }^{2}}-\rho_{\text {rich }} g\left(H+Z_{g e n}\right)+\frac{\dot{m}_{r i c h}{ }^{2}}{A_{g e n}{ }^{2}} \cdot\left(\frac{1}{\rho_{I P}}-\frac{1}{\rho_{\text {rich }}}\right)+\rho_{I P} \cdot g \cdot\left(Z_{g e n}+L\right)+=0
$$


The submergence ratio can be thus expressed as:

$$
\left.\frac{\left(H+Z_{g e n}\right)}{\left(Z_{g e n}+L\right)}=\frac{\dot{m}_{r i c h}{ }^{2}}{\rho_{\text {rich }} \cdot g \cdot\left(Z_{\text {gen }}+L\right)} \mid \frac{1}{2 \rho_{\text {rich }} \cdot A_{D C}{ }^{2}}+\frac{1}{A_{g e n}{ }^{2}} \cdot\left(\frac{1}{\rho_{T P}}-\frac{1}{\rho_{\text {rich }}}\right)\right\rfloor+\frac{\rho_{T P}}{\rho_{\text {rich }}}
$$

Still for a fixed submergence ratio there are two unknown parameter $\quad \dot{m}_{\text {rich }}$ the mass flow rate of the rich solution and $\dot{m}_{r e f}$ the mass flow rate of the refrigerant.

Let us express the mass flow rate of the refrigerant as a function of the inlet heat input. To do so let us apply heat balance on the generator with assumption that the inlet temperature to the generator is equal to the reservoir temperature. The supplied heat input $\dot{W}$ heats the rich solution in the generator increasing its temperature from the equilibrium temperature $T_{\text {res }}$ to the generator temperature $T_{\text {gen }}$ :

$$
\dot{W}=\dot{m}_{n c h} \cdot \bar{c}_{p}\left(T_{g e n}-T_{r e s}\right)
$$

The heat capacity was calculated based on [17] experimental results.

Comparison between the experimental and the theoretical results is plotted in Fig .2. It can be seen from the figure that the higher the flow rates the more accurate is the model. A possible reason for the differences between the experimental and the theoretical results is the heat losses due to back absorption at low heat inputs.

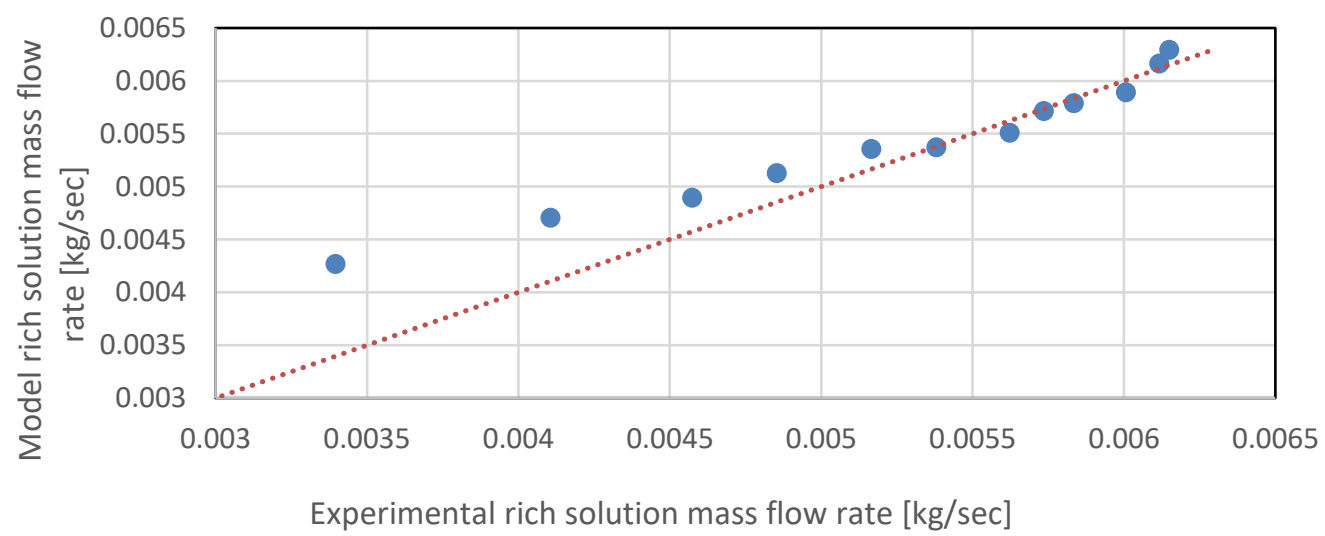

Fig. 2: Calculated rich solution mass flow rate based on the model in eq. 31 vs. the experimental results of [8].

\subsection{Comparison with the Results}

The experimental results were plotted against the experimental results of [8]. It can be seen from fig. 3 that the current model that uses the drift flux model with the assumption of laminar flow fits more accurately to the experimental results. 


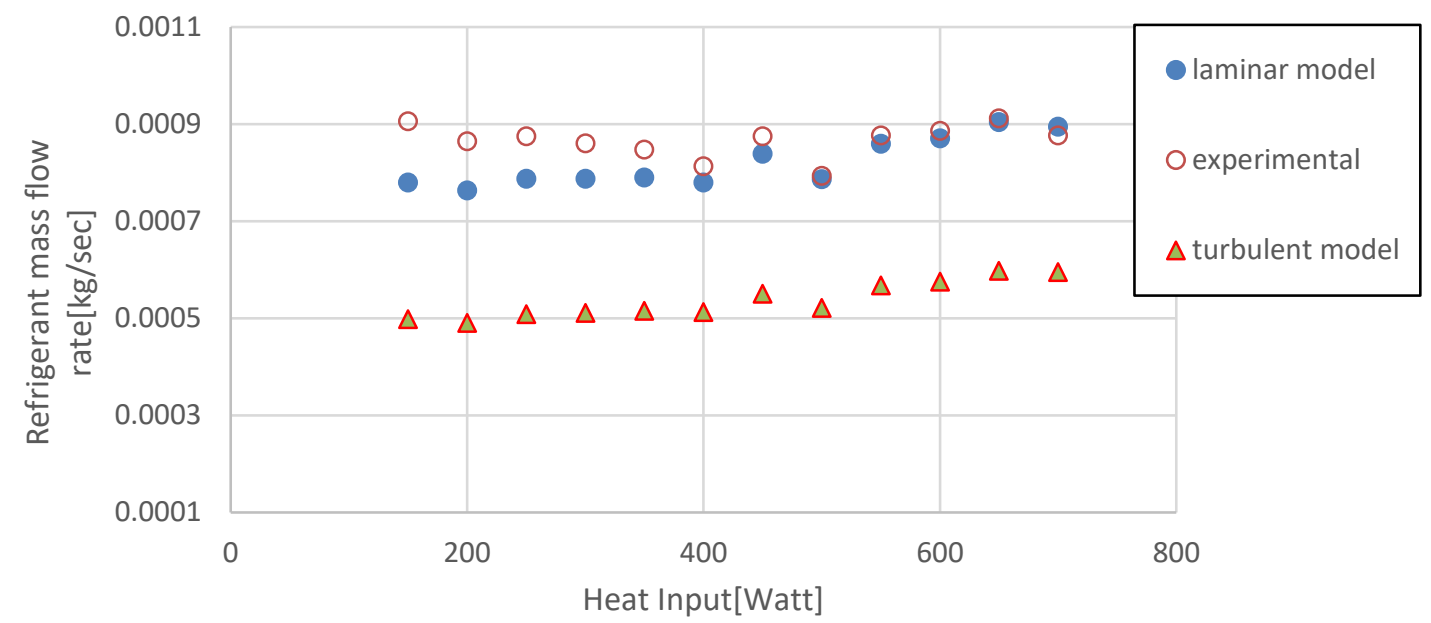

Fig. 3: Separated mass flow rate of the refrigerant vs. heat input for experimental results, model with laminar flow assumption and model with turbulent flow assumption.

Reynolds numbers were calculated and plotted vs. heat power increase for the refrigerant, poor solution and the for the mixture (Fig. 4). While the poor solution and the mixture were in laminar flow regime, the refrigerant was in transient regime.

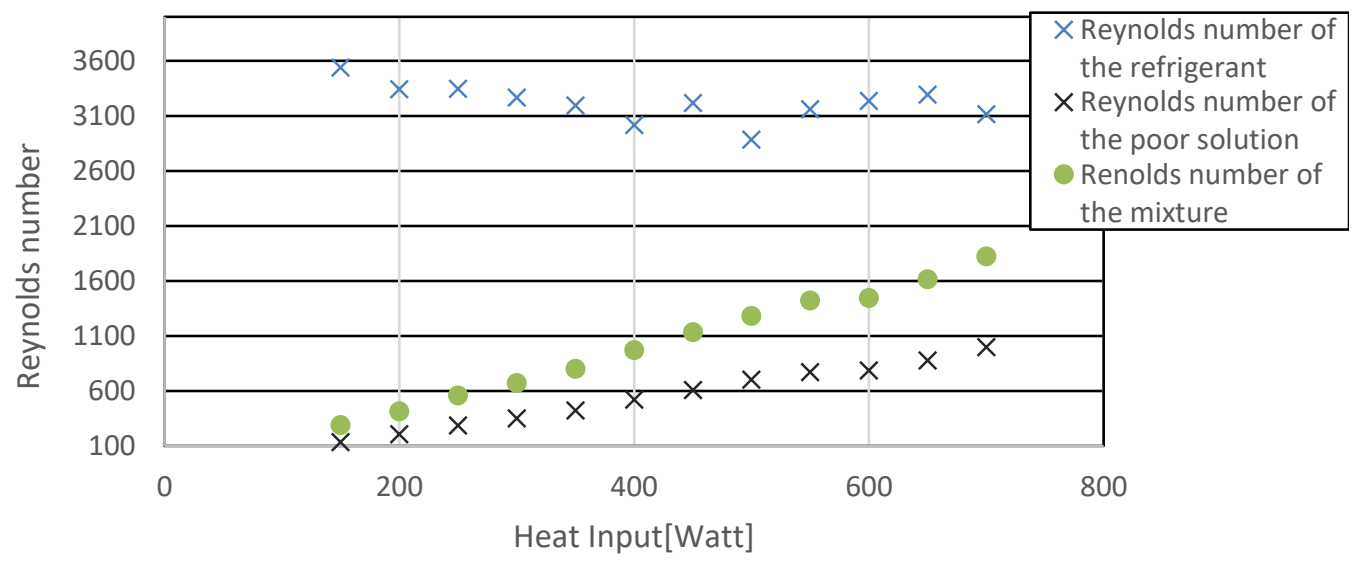

Fig. 4: Reynolds numbers of the refrigerant, the poor solution and the mixture vs. heat input.

\section{Conclusions}

A theoretical model based on the model of Stenning and Martin was presented. The presented model uses drift flux model together with laminar flow assumption. Experimental results show that the flow of the poor solution (the liquid phase) is indeed laminar. The model allows to predict the amounts of the separated refrigerant for a certain value of submergence value. In addition, the present model correlates between the amount of the applied heat and the rich solution mass flow rate. It is important to state, that the previous models were developed for an adiabatic ail-water lift tubes where heat did not play any significant role. The present model correlates the geometrical and the operational parameters of the bubble pump and fits the experimental results.

\section{References}

[1] D. J. Nicklin, “The airlift pump: theory and optimization," Trans. Inst. Chem. Eng., vol. 41, pp. 29-39, 1963.

[2] H. Stenning and C. B. Martine, "An Analytical and experimental study of airlift pump performance," ASME J. Eng. for Pow., vol. 90, pp. 106-112, 1968. 
[3] A. D. Delano, "Design analysis of the Einstein refrigeration cycle Robot Vision," Ph.D. dissertation, Georgia Institute of Technology, 1998.

[4] A. Sathe, "Experimental and Theoretical Studies on a Bubble Pump for a Diffusion-Absorption Refrigeration System," Master's thesis, Indian Institute of Technology Madras and University of Stuttgart, 2001.

[5] D. Chisholm, Two-phase flow in pipelines and heat. New York: George Goodwin, 1983.

[6] A. Koyfman, M. Jelinek, A. Levy and I. Borde, "An experimental investigation of bubble pump performance for diffusion absorption refrigeration system with organic working fluids," Applied Thermal Engineering, pp. 1881-1894, 2003.

[7] A. W. S. Shihab, and A. M. A. Morad, "Experimental Investigation of Water Vapor- Bubble pump Characteristics and its Mathematical Model Reconstruction," Eng. \& Tech. Journal, vol. 30, No.11, pp. 1870- 1885, 2012.

[8] B. Gurevich, M. Jelinek, A. Levy and I. Borde, "Performance of a set of parallel bubble pumps operating with a binary solution of R134a-DMAC", Applied Thermal Engineering, vol. 75, pp. 724-730, 2015.

[9] N. Zuber. And J. Findlay, "Average volumetric concentration in two-phase flow system," Trans. ASME Journal of Heat Transfer, vol. 87, pp. 453-468, 1965.

[10] G. B. Wallis, One-dimensional two-phase Flow. McGraw-Hill, 1969.

[11] M. Ishii, "One-dimensional drift-flux model and constitutive equations for relative motion between phase in various two-phase flow regimes," Argonne Nat. Lab. ANL-77-47, 1977.

[12] R. Collins, F. F. de Moraes, J. F. Davidson and D. Harrison, "The motion of a large gas bubble rising through liquid flowing in a tube," J. Fluid Mech., vol. 89, pp. 497-514, 1978.

[13] J. R. Grace, R. Clift, "Dependence of slug rise velocity on tube Reynolds number in vertical gas liquid flow," Chem. Eng. Sci., vol. 34, pp. 1348-1350, 1979.

[14] K. Bendiksen, "On the motion of long bubbles in vertical tubes," Int. J. Multiphase Flow, vol. 11, pp. 797-812, 1985.

[15] R. H. Bonnecaze, W. Eriskine. Jr and E. J. Greskovich, "Holdup and pressure drop for two-phase slug flow in inclined pipelines," AIChE J., vol. 17, pp. 1109-1113, 1971.

[16] I. Borde, M. Jelinek and N. C. Daltrophe, "Development of Advanced Absorption Systems Driven by Low Temperature Heat Sources," Int. J. Refrigeration, vol. 31, pp. 521-530, 1993.

[17] A. Yokozeki, "Theoretical performances of various refrigerant-absorbent pairs in a vapor-absorption refrigeration cycle by the use of equations of state," App. Energy, vol. 80, no. 4, pp. 383-399, 2005. 